JOURNAL OF SYNCHROTRON RADIATION

ISSN 1600-5775

\section{X-ray radiation damage to biological samples: recent progress}

\author{
Elspeth F. Garman ${ }^{\mathrm{a} *}$ and Martin Weik ${ }^{\mathrm{b}}$ \\ aDepartment of Biochemistry, University of Oxford, South Parks Road, Oxford OX1 3QU, UK, and \\ bUniv. Grenoble Alpes, CEA, CNRS, Institut de Biologie Structurale, F-38044 Grenoble, France. \\ *Correspondence e-mail: elspeth.garman@bioch.ox.ac.uk
}

Keywords: X-ray radiation damage; macromolecular crystallography; dose; X-ray imaging; SAXS; DNA; temperature effects; metalloproteins.

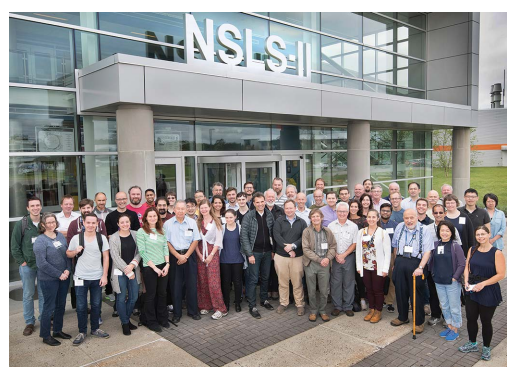

Nearly 20 years of research into radiation damage effects during macromolecular crystallography (MX) experiments carried out at both cryo and room temperatures has given us the tools to recognize the symptoms and quantify the effects of this damage. It is well established that protein crystals suffer both global and site-specific structural damage to particular amino acids in a reproducible order during X-ray exposure, and these effects have now been thoroughly characterized in a large variety of samples. However, there are still aspects of the phenomenon that bear closer scrutiny, especially with the recent commissioning of several MX new synchrotron beamlines which provide ever higher flux densities. Many radiation damage investigations have thus far been reported in the literature (see for example the special issues of the Journal of Synchrotron Radiation arising from talks and posters given at the 2nd to 9th International Workshops on Radiation Damage to Biological Crystalline Samples, published in 2002, 2005, 2007, 2009, 2011, 2013, 2015 and 2017).

In this special issue of the Journal of Synchrotron Radiation there are nine papers on various aspects of radiation damage in MX and small-angle X-ray scattering (SAXS) experiments. 
Much of the research reported in them was presented and/or discussed at the 10th International Workshop on Radiation Damage to Biological Samples held at NSLS II at Brookhaven National Laboratory in September 2018. The papers encompass a wide range of disparate investigations on a variety of samples.

During the last 20 years, cryocrystallographic methods have been very widely utilized in MX to reduce the rate of radiation damage to the samples, but increasing numbers of experiments are now being carried out at room temperature (RT) using serial crystallographic methods, including those carried out at $\mathrm{X}$-ray free-electron laser (XFEL) sources. Studying the same structures at different temperatures can give new valuable information in physiologically relevant regimes, including dynamical processes, otherwise hidden residue conformations (Fraser et al., 2011) and, for metallo-enzymes, on redox states.

Here (Ueno et al., 2019), a helical data collection strategy at an incident energy of $30 \mathrm{keV}$ from nine large crystals of cytochrome $c$ oxidase at $100 \mathrm{~K}$ enabled a low-dose $(58 \mathrm{kGy}$ diffraction weighted dose, DWD; Zeldin et al., 2013a) structure to be derived, allowing detailed comparison with the damage-free XFEL-determined structure (Hirata et al., 2014). The peroxide ligand bond lengths were identical in the two structures, although, from analysis of residual electron density maps, there was evidence of low levels of specific damage in the structure derived from the synchrotron data. The redox states of the heme metal in the protein were monitored using UV-Vis absorption spectroscopy carried out at incident X-ray energies of $10,13.8$ and $17.7 \mathrm{keV}$, but no energy dependence of specific damage was detected.

For the incident energy of $30 \mathrm{keV}$ used in the above study, a pixel detector with a cadmium telluride (CdTe) sensor was used, as these have a much higher detective quantum efficiency than silicon sensors as the X-ray energy increases. By means of simulations, another paper in this issue addresses the potential gains in using higher incident X-ray energies when using a CdTe detector. The optimum energy for MX experiments has long been debated (Arndt, 1984; Helliwell \& Fourme, 1983; Paithankar \& Garman, 2010; Fourme et al., 2012), and the dependence of damage on incident energy has been experimentally investigated by a number of groups $(e . g$. Shimizu et al., 2007; Weiss et al., 2005; Homer et al., 2011; Liebschner et al., 2015) with varying conclusions on the specific damage rates at $100 \mathrm{~K}$, but a consensus that global damage rates as a function of dose seem to be independent of incident energy has been reached. Reported here are the results of Monte Carlo simulations of the diffraction efficiency (DE, ratio of diffraction to absorbed dose) as a function of energy (Dickerson \& Garman, 2019). These calculations take into account the energy response of the CdTe detector as well as the probability of photoelectron escape from the irradiated crystal volume and entry of photoelectrons from any irradiated surrounding material. As has previously been suggested, photoelectron escape can potentially reduce the absorbed dose (Nave \& Hill, 2005; Cowan \& Nave, 2008; Bury et al., 2018; Marman et al., 2018) and thus improve the DE. The results presented here identify a maximum in the DE at a 'sweet spot' of $26 \mathrm{keV}$ if using crystals of $5 \mu \mathrm{m}$ or less with a microbeam which is either matched in size to (or smaller than) the crystal. Under these conditions more than a factor of two improvement over the DE at $12.4 \mathrm{keV}$ can be obtained.

The danger of drawing mechanistic conclusions from structural studies of enzymes is highlighted by the results reported for seven $100 \mathrm{~K}$ xylose isomerase structures at DWDs ranging between 0.13 and $3.88 \mathrm{MGy}$ (Taberman et al., 2019). As well as examining the usual global metrics and electron density difference maps, the analysis includes the use of a new metric, $B_{\text {net }}$, calculated using the program $R A B D A M$ (Shelley et al., 2018), now incorporated into the CCP4 suite (Winn et al., 2011). This metric aims to provide a 'damage index' for $100 \mathrm{~K}$ structures, and is derived from the atomic $B$ values of the structure, from which the packing density has been deconvoluted. Xylose isomerase is an important enzyme industrially, and a detailed knowledge of its mechanism of action could aid its use in biotechnology but a full description has long been elusive. It has two divalent metal cofactors, one structural and one catalytic. The latter is thought to adopt three different positions during the reaction cycle. The absolute positions of these atoms changed with increasing dose, but when these shifts were corrected for the radiation-induced unit cell expansion it was clear that there was no relative movement. It is thus possible that radiation chemistry effects impacting structure determination may impact some of the various mechanistic conclusions drawn in the literature for this enzyme (Asboth \& Naray-Szabo, 2000; Fenn et al., 2004; Munshi et al., 2014).

Indeed, radiation chemistry may be able to explain the details of the observed specific damage progression in MX at $100 \mathrm{~K}$. Close \& Bernhard ${ }^{\mathbf{1}}$ (2019) provide an interesting discussion of a computational description of a complete mechanism for oxidative and reductive two-electron (or two sequential one-electron) interconversions. The investigation steps through each of the amino acids expected to be most sensitive to these redox processes, including cysteine, aspartate and glutamate, tyrosine, cysteine, and methionine, aiming to provide a comprehensive picture of eventual outcomes in each case. An explanation for the apparent radiation stability of the peptide backbone is also offered. The work is informed by previous experimental low-temperature electron paramagnetic resonance results, for example as in D'Arcy \& Sevilla (1979), and expands on ideas first discussed by the late Bill Bernhard.

Such calculations can inform the radiation chemistry of ensembles with limited numbers of atoms (depending on the computational methods employed and the computational resources available), such as those investigated by smallmolecule crystallographers (SMX). With the increased flux densities provided by modern SMX synchrotron beamlines, radiation damage to the investigated samples is now increasingly being observed (Morgan et al., 2018). The first systematic investigation of the effect of sample temperature $(30,60,100$ and $120 \mathrm{~K}$ ) and four different dose rates on rates of global and

\footnotetext{
1 Deceased.
} 
specific radiation damage to crystals of one species of small molecule has recently been reported (Christensen et al., 2019). The results indicate that, as in MX, the resulting structures can be modified by radiation damage effects, and that an awareness of this phenomenon among experimenters would be instructive for structure interpretation. For this study, the MX dose estimation software RADDOSE-3D (Zeldin et al., 2013b) was modified for use in SMX.

Moving back to MX, as the review by van den Bedem \& Wilson (2019) demonstrates, studies of the progression of radiation damage inflicted by X-rays on cysteines within proteins can be linked to understanding global protein conformational changes and function, and can give valuable insights into biological mechanism rather than confounding it. In MX, the frequently observed reduction of disulfide bonds to ionized thiolates $\left(\mathrm{Cys}_{-} \mathrm{S}^{-}\right)$with dose can change the local electrostatic environment within the protein, since these are highly reactive species. If the cysteine is near the active site, the $\mathrm{p} K_{\mathrm{a}}$ value of the cysteine thiol sidechain will be affected, and the covalent modification alters the local protein dynamics, potentially impacting rational drug discovery efforts. The authors give a detailed example of this, describing experiments which reveal catalysis mechanism by using sitespecific cysteine photoreduction and temperature-controlled MX in isocyanide hydrolase at both $100 \mathrm{~K}$ (Lakshminarasimhan et al., 2010) and RT (274-277 K) (DasGupta et al., 2019). These studies were complemented computationally by molecular dynamics simulations which gave further insights into conformational changes. However, a note of caution is sounded that biochemical validation of the effects of cysteine modification is essential before drawing reliable mechanistic conclusions from MX data.

Another account of the biologically enlightening knowledge to be gained from a study of radiation damage effects is given by Stachowski et al. (2019). A RT low-dose (14.2 Gy) SAXS study on solutions of transforming growth factor beta-1 (TGF $\beta$-1), a potent cytokine, caged by latency associated peptide (LAP) is presented, along with additional information obtained from CD spectroscopy. A dose as low as $5 \mathrm{~Gy}$ from $\mathrm{X}$-ray irradiation is known to induce the dissociation of the LAP and lead to subsequent activation of TGF $\beta-1$, with resulting impacts on human health. However, the results reported here suggest that although this activation is initiated by the irradiation it is not driven by it. An extended conformation of unbound LAP was detected, indicating a structural transition during TGF $\beta$ - 1 activation. Low doses of therapeutic radiation can thus cause structural impacts. The methods discussed offer pathways to understand these structural changes aided by knowledge of the radiation damage process. Similarly, a recent time-resolved synchrotron SAXS study provided evidence for radiation-induced domain swapping in human cystatin C (Taube et al., 2019).

SAXS was also used, but in scanning mode (sSAXS), to analyse the parameters affecting radiation damage rates in RT cardiac muscle cells and tissue (Nicolas et al., 2019), by monitoring the intensity of the reflection from the acto-mysin lattice following microbeam $(<3 \mu \mathrm{m})$ irradiation. The vari- ables investigated included the scan step size, the length of the horizontal scan, the vertical distance between scan lines and the exposure time per scan point. Optimum values were determined for all four parameters. The accompanying Monte Carlo simulations of the dose distributions resulting from these beams on soft muscle tissue allow for more informed planning of future experiments.

For radiation damage rates to be reduced in cryocrystallography, it is vital that the crystal be kept below the temperature at which $\mathrm{OH}$ radicals are thought to become increasingly mobile (between 115 and $130 \mathrm{~K}$; Owen et al., 2012). Some of the energy lost by the X-ray beam in the crystal through inelastic processes (the photoelectric and Compton effects) is converted into heat, and this causes the crystal temperature to rise. Quantifying this effect has been very challenging for the field, since temperature-gauging devices take heat into the system and affect the results. Previous measurements with a thermal imaging camera at a third-generation synchrotron with an uncollimated FWHM $0.103 \mathrm{~mm}$ (horizontal) and $0.084 \mathrm{~mm}$ (vertical) beam at $6.5 \mathrm{keV}$ incident X-ray energy, a flux of $3.24 \times 10^{12}$ photons s$^{-1}$, and using $1 \mathrm{~mm}$ and $2 \mathrm{~mm}$ glass beads held in a $100 \mathrm{~K}$ nitrogen stream, showed a $10-15 \mathrm{~K}$ temperature rise (Snell et al., 2007). In this issue, Warren et al. (2019) use the previously well characterized fluorescence induced in 20 and $40 \mu \mathrm{m}$ ruby crystals to measure the temperature rise from $100 \mathrm{~K}$ caused by $20 \mu \mathrm{m} \times 20 \mu \mathrm{m} 9 \mathrm{keV}$ and $12.8 \mathrm{keV}$ X-ray beams with fluxes of $3.18 \times 10^{12}$ (dose rate $7.4 \mathrm{MGy} \mathrm{s}^{-1}$ ) and $1.19 \times 10^{12}$ photons s$^{-1}$ (dose rate $1.5 \mathrm{MGy} \mathrm{s}^{-1}$ ), respectively. Fluorescence was also induced by a $532 \mathrm{~nm}$ laser to provide calibration data of wavelength against temperature. For typical MX data collection conditions (crystal sizes, beam energies, flux densities and dose rate $<5 \mathrm{MGy} \mathrm{s}^{-1}$ ) the temperature rise was found to be around $20 \mathrm{~K}$. Clearly, for higher flux densities, heating is likely to be significant and will allow increased mobility of radicals, with potentially deleterious effects on crystal lifetimes in the beam, but perhaps mitigated by the presence of scavenging agents in the cryobuffers.

Previous MX studies (Bury et al., 2015, 2016) have concluded that, compared with those of proteins, crystals of both DNA-protein and RNA-protein complexes at $100 \mathrm{~K}$ appear to be much more robust in terms of specific structural damage. This has raised the question as to whether the oligonucleotides are protected by the scavenging action of the protein or if they are intrinsically less susceptible to radiation damage. Bugris et al. (2019) present a study of the degradation with dose of a crystal of a 16-mer DNA at $100 \mathrm{~K}$, for which they analyse both global and specific damage indicators. For the latter, the software tool RIDL (Radiation-Induced Density Loss; Bury \& Garman, 2018) was utilized to allow objective inspection of the electron density loss with dose up to 35 MGy. Of the four nucleotide species, guanine appeared to be the most sensitive to specific damage, and differential damage rates of the guanines in the sequence were noted, as with susceptible amino acids in proteins. The damage was generally more severe in the vicinity of some (but not all) of the bound calcium ions, and phosphate moieties showed $\mathrm{P}-\mathrm{O}$ and $\mathrm{C}-\mathrm{O}$ 
bond breaks. The authors conclude that the previously observed radiation hardness of the DNA and RNA in complex with protein is likely to be a result of scavenging action by the protein.

Using serial crystallography or multi-crystal data collection methods at synchrotron sources, the total absorbed dose per data set is distributed over a large number of crystals, and radiation damage thus minimized, but not eliminated. Carried out at an XFEL, so-called serial femtosecond crystallography (SFX) generally provides structures free of secondary radiation damage, because of diffraction from the femtosecond pulses outrunning destruction (Neutze et al., 2000). Yet, higher-intensity longer XFEL pulses can generate damage [reviewed by Nass (2019)]. Two recent studies combined dose-dependent crystallography at synchrotrons with SFX at XFELs. Tosha and co-workers (Tosha et al., 2017) collected cryo-crystallographic data on macrocrystals of the enzyme nitric oxide reductase and analysed the $\mathrm{Fe}-\mathrm{N}-\mathrm{O}$ coordination geometry at the heme group. The SFX structure showed a slight $\mathrm{Fe}-\mathrm{N}-\mathrm{O}$ bending, characteristic of the undamaged state that markedly increased as a function of dose in the synchrotron structures. Similar results were obtained by RT serial crystallography on heme peroxidase microcrystals, where a functionally relevant bond length linearly increased as a function of dose in the synchrotron structures. Extrapolation back to zero-dose predicted the bond length of a radiationdamage-free structure that has been experimentally found in the SFX structure (Ebrahim et al., 2019a).

Serial or multi-crystal synchrotron crystallography (SSX) can provide valuable new insight into RT radiation damage. Warkentin and co-workers have shown that non-uniform illumination with a Gaussian microbeam can explain the observed non-exponential diffraction-intensity decay as a function of dose (Warkentin et al., 2017). A radiation-induced exchange between two polymorphs with different unit-cell parameters has been observed in another serial crystallography study (Ebrahim et al., 2019b). Analysis of multicrystal synchrotron structures showed that global and specific radiation damage occur at similar rates at $\mathrm{RT}$, but not at $100 \mathrm{~K}$ (Gotthard et al., 2019). The imminent increase of fourthgeneration synchrotron beamlines will certainly generate many more opportunities for furthering our understanding of radiation damage at RT through existing and future serial- and multi-crystal data collection methods.

It has been shown recently (Foos et al., 2018) that SSX can also be used at $100 \mathrm{~K}$ to determine structures by radiationdamage-induced phasing (RIP). In RIP, a low-dose and then a high-dose dataset are collected from the same crystal, usually with a controlled X-ray or UV induced 'burn' in between. The two datasets are compared, and the difference in signal from (for instance) the sulfur atoms in disulfide bonds, which preferentially break and disorder during X-ray/UV exposure, can be used to obtain starting phases for structure determination (Ravelli et al., 2003). In a proof-of-principle experiment, Foos et al. collected data at $100 \mathrm{~K}$ from many microcrystals of two different test proteins (insulin and thaumatin), using both X-ray and UV 'burn' protocols. They investigated how the success of structure solution depended on both the multiplicity of the data and the dose difference between the two datasets, and found that, for X-ray RIP, substructures derived from the RIP signal could be improved up to $4 \mathrm{MGy}$ and that, up to a certain point, higher multiplicity (8-fold for thaumatin and 25-fold for insulin) increased the RIP peak heights. This level of multiplicity for RIP can only be obtained by merging data from many crystals, as in SSX methods.

Current advances in the resolution of macromolecules now obtainable using electron microscopes have allowed observation of specific electron radiation damage in several studies. A systematical analysis of cryo-electron diffraction data from nanocrystals (MicroED) of proteinase $\mathrm{K}$ (at $2 \AA$ ) and a hexapeptide (at $1 \AA$ ) at two different electron fluxes has been carried out (Bartesaghi et al., 2018). The diffraction intensities decreased exponentially with exposure. Similar specific damage signatures were observed to those that are well established in MX. Even at very low exposures of around $0.1 \mathrm{e}^{-} \AA^{-2}$, disulfide bonds in proteinase $\mathrm{K}$ were affected, and decarboxylation of acidic side chains was seen at around $2 \mathrm{e}^{-} \AA^{-2}$.

The detailed study of radiation damage in biological samples over the past two decades has resulted in many practical developments that have helped the field and illustrated some of the complexities of the problem. It has extended beyond single crystal data collection with the phenomenon impacting biological imaging in many areas. The field has extended with the development of new sources and growing understanding of the process, mitigation, and use of the phenomena. The detailed exploration of radiation damage in the study of biological samples still has much to teach us. To this extent, an 11th Workshop on this topic will be held at the Swiss Light Source in 2020.

\section{Acknowledgements}

We thank Ian Carmichael and Edward Snell for their critical reading of this manuscript. The 10th International Workshop on Radiation Damage to Crystalline Biological Samples, at which most of the work in this special issue was presented, would not have been possible without the invaluable support of NSLS II who hosted it, and the hard work of the Local Organizing Committee of Martin Fuchs, Jean Jakoncic, Sean McSweeney and Mercy Baez. We are most grateful to them for their enthusiasm and efforts.

\section{References}

Arndt, U. W. (1984). J. Appl. Cryst. 17, 118-119.

Asboth, B. \& Naray-Szabo, G. (2000). Curr. Protein Pept. Sci. 1, $237-$ 254.

Bartesaghi, A., Aguerrebere, C., Falconieri, V., Banerjee, S., Earl, L. A., Zhu, X., Grigorieff, N., Milne, J. L. S., Sapiro, G., Wu, X. \& Subramaniam, S. (2018). Structure, 26, 848-856.

Bedem, H. van den \& Wilson, M. A. (2019). J. Synchrotron Rad. 26, 958-966.

Bugris, V., Harmat, V., Ferenc, G., Brockhauser, S., Carmichael, I. \& Garman, E. F. (2019). J. Synchrotron Rad. 26, 998-10009. 
Bury, C., Garman, E. F., Ginn, H. M., Ravelli, R. B. G., Carmichael, I., Kneale, G. \& McGeehan, J. E. (2015). J. Synchrotron Rad. 22, 213224.

Bury, C. S., Brooks-Bartlett, J. C., Walsh, S. P. \& Garman, E. F. (2018). Protein Sci. 27, 217-228.

Bury, C. S. \& Garman, E. F. (2018). J. Appl. Cryst. 51, 952-962.

Bury, C. S., McGeehan, J. E., Antson, A. A., Carmichael, I., Gerstel, M., Shevtsov, M. B. \& Garman, E. F. (2016). Acta Cryst. D72, 648657.

Christensen, J., Horton, P. N., Bury, C. S., Dickerson, J. L., Taberman, H., Garman, E. F. \& Coles, S. J. (2019). IUCrJ, 6, 703-713.

Close, D. \& Bernhard, W. A. (2019). J. Synchrotron Rad. 26, 945-957. Cowan, J. A. \& Nave, C. (2008). J. Synchrotron Rad. 15, 458-462.

D’Arcy, J. B. \& Sevilla, M. D. (1979). Radiat. Phys. Chem. 13, 119-126.

DasGupta, M., Budday, D., Madzelan, P., Seravalli, J., Hayes, B., Sierra, R. G., Alonso-Mori, R., Brewster, A. S., Sauter, N. K., Applegate, G. A., Tiwari, V., Berkowitz, D. B., Thompson, M. C., Fraser, J. S., Wall, M. E., van den Bedem, H. \& Wilson, M. A. (2019). bioRxiv: 524751.

Dickerson, J. L. \& Garman, E. F. (2019). J. Synchrotron Rad. 26, $922-$ 930.

Ebrahim, A., Appleby, M. V., Axford, D., Beale, J., Moreno-Chicano, T., Sherrell, D. A., Strange, R. W., Hough, M. A. \& Owen, R. L. (2019b). Acta Cryst. D75, 151-159.

Ebrahim, A., Moreno-Chicano, T., Appleby, M. V., Chaplin, A. K., Beale, J. H., Sherrell, D. A., Duyvesteyn, H. M. E., Owada, S., Tono, K., Sugimoto, H., Strange, R. W., Worrall, J. A. R., Axford, D., Owen, R. L. \& Hough, M. A. (2019a). IUCrJ, 6, 543-551.

Fenn, T. D., Ringe, D. \& Petsko, G. A. (2004). Biochemistry, 43, 64646474.

Foos, N., Seuring, C., Schubert, R., Burkhardt, A., Svensson, O., Meents, A., Chapman, H. N. \& Nanao, M. H. (2018). Acta Cryst. D74, 366-378.

Fourme, R., Honkimäki, V., Girard, E., Medjoubi, K., Dhaussy, A.-C. \& Kahn, R. (2012). J. Appl. Cryst. 45, 652-661.

Fraser, J. S., van den Bedem, H., Samelson, A. J., Lang, P. T., Holton, J. M., Echols, N. \& Alber, T. (2011). Proc. Natl Acad. Sci. USA, 108, 16247-16252.

Gotthard, G., Aumonier, S., De Sanctis, D., Leonard, G., von Stetten, D. \& Royant, A. (2019). IUCrJ, 6, 665-680.

Helliwell, J. R. \& Fourme, R. (1983). The ESRF as a Facility for Protein Crystallography: Report and Design Study. ESRP Report IRI-4/83, pp. 1-36. European Science Foundation, Strasbourg, France.

Hirata, K., Shinzawa-Itoh, K., Yano, N., Takemura, S., Kato, K., Hatanaka, M., Muramoto, K., Kawahara, T., Tsukihara, T., Yamashita, E., Tono, K., Ueno, G., Hikima, T., Murakami, H., Inubushi, Y., Yabashi, M., Ishikawa, T., Yamamoto, M., Ogura, T., Sugimoto, H., Shen, J. R., Yoshikawa, S. \& Ago, H. (2014). Nat. Methods, 11, 734-736.

Homer, C., Cooper, L. \& Gonzalez, A. (2011). J. Synchrotron Rad. 18, 338-345.

Lakshminarasimhan, M., Madzelan, P., Nan, R., Milkovic, N. M. \& Wilson, M. A. (2010). J. Biol. Chem. 285, 29651-29661.

Liebschner, D., Rosenbaum, G., Dauter, M. \& Dauter, Z. (2015). Acta Cryst. D71, 772-778.
Marman, H., Darmanin, C. \& Abbey, B. (2018). Crystals, 8, 267.

Morgan, L. C. F., Kim, Y. B., Blandy, J. N., Murray, C. A., Christensen, K. E. \& Thompson, A. L. (2018). Chem. Commun. 54, 9849-9852.

Munshi, P., Snell, E. H., van der Woerd, M. J., Judge, R. A., Myles, D. A. A., Ren, Z. \& Meilleur, F. (2014). Acta Cryst. D70, 414-420. Nass, K. (2019). Acta Cryst. D75, 211-218.

Nave, C. \& Hill, M. A. (2005). J. Synchrotron Rad. 12, 299-303.

Neutze, R., Wouts, R., van der Spoel, D., Weckert, E. \& Hajdu, J. (2000). Nature, 406, 752-757.

Nicolas, J.-D., Aeffner, S. \& Salditt, T. (2019). J. Synchrotron Rad. 26, 980-990.

Owen, R. L., Axford, D., Nettleship, J. E., Owens, R. J., Robinson, J. I., Morgan, A. W., Doré, A. S., Lebon, G., Tate, C. G., Fry, E. E., Ren, J., Stuart, D. I. \& Evans, G. (2012). Acta Cryst. D68, 810 818.

Paithankar, K. S. \& Garman, E. F. (2010). Acta Cryst. D66, 381-388.

Ravelli, R. B., Leiros, H. K., Pan, B., Caffrey, M. \& McSweeney, S. (2003). Structure, 11, 217-224.

Shelley, K. L., Dixon, T. P. E., Brooks-Bartlett, J. C. \& Garman, E. F. (2018). J. Appl. Cryst. 51, 552-559.

Shimizu, N., Hirata, K., Hasegawa, K., Ueno, G. \& Yamamoto, M. (2007). J. Synchrotron Rad. 14, 4-10.

Snell, E. H., Bellamy, H. D., Rosenbaum, G. \& van der Woerd, M. J. (2007). J. Synchrotron Rad. 14, 109-115.

Stachowski, T., Grant, T. D. \& Snell, E. H. (2019). J. Synchrotron Rad. 26, 967-979.

Taberman, H., Bury, C. S., van der Woerd, M. J., Snell, E. H. \& Garman, E. F. (2019). J. Synchrotron Rad. 26, 931-944.

Taube, M., Pietralik, Z., Szymanska, A., Szutkowski, K., Clemens, D., Grubb, A. \& Kozak, M. (2019). Sci. Rep. 9, 8548.

Tosha, T., Nomura, T., Nishida, T., Saeki, N., Okubayashi, K., Yamagiwa, R., Sugahara, M., Nakane, T., Yamashita, K., Hirata, K., Ueno, G., Kimura, T., Hisano, T., Muramoto, K., Sawai, H., Takeda, H., Mizohata, E., Yamashita, A., Kanematsu, Y., Takano, Y., Nango, E., Tanaka, R., Nureki, O., Shoji, O., Ikemoto, Y., Murakami, H., Owada, S., Tono, K., Yabashi, M., Yamamoto, M., Ago, H., Iwata, S., Sugimoto, H., Shiro, Y. \& Kubo, M. (2017). Nat. Commun. 8, 1585

Ueno, G., Shimada, A., Yamashitac, E., Hasegawa, K., Kumasaka, T., Shinzawa-Itoh, K., Yoshikawa, S., Tsukihara, T. \& Yamamoto, M. (2019). J. Synchrotron Rad. 26, 912-921.

Warkentin, M. A., Atakisi, H., Hopkins, J. B., Walko, D. \& Thorne, R. E. (2017). IUCrJ, 4, 785-794.

Warren, A. J., Axford, D. \& Owen, R. L. (2019). J. Synchrotron Rad. 26, 991-997.

Weiss, M. S., Panjikar, S., Mueller-Dieckmann, C. \& Tucker, P. A. (2005). J. Synchrotron Rad. 12, 304-309.

Winn, M. D., Ballard, C. C., Cowtan, K. D., Dodson, E. J., Emsley, P., Evans, P. R., Keegan, R. M., Krissinel, E. B., Leslie, A. G. W., McCoy, A., McNicholas, S. J., Murshudov, G. N., Pannu, N. S., Potterton, E. A., Powell, H. R., Read, R. J., Vagin, A. \& Wilson, K. S. (2011). Acta Cryst. D67, 235-242.

Zeldin, O. B., Brockhauser, S., Bremridge, J., Holton, J. M. \& Garman, E. F. (2013a). Proc. Natl Acad. Sci. USA, 110, 20551-20556.

Zeldin, O. B., Gerstel, M. \& Garman, E. F. (2013b). J. Synchrotron Rad. 20, 49-57. 